\title{
A Gaia DR2 search for dwarf galaxies towards Fermi-LAT sources: implications for annihilating dark matter
}

\author{
Ioana Ciucă ${ }^{1,2 \star}$, Daisuke Kawata ${ }^{1}$, Shin'ichiro Ando ${ }^{3,4}$, Francesca Calore ${ }^{5}$, Justin \\ I. Read ${ }^{6}$ and Cecilia Mateu ${ }^{7}$ \\ ${ }^{1}$ Mullard Space Science Laboratory, University College London, Holmbury St. Mary, Dorking, Surrey, RH5 6NT, UK \\ 2 LSSTC Data Science Fellow \\ ${ }^{3}$ GRAPPA Institute, University of Amsterdam, 1098 XH Amsterdam, Netherlands \\ ${ }^{4}$ Kavli Institute for the Physics and Mathematics of the Universe, University of Tokyo, Kashiwa 277-8583, Japan \\ 5 Univ. Grenoble Alpes, USMB, CNRS, LAPTh, F-74940 Annecy, France \\ ${ }^{6}$ Department of Physics, University of Surrey, Guildford, GU2 7XH, UK \\ 7 Centro de Investigaciones de Astronomía, AP 264, Mérida 5101-A, Venezuela
}

Accepted 2018 July 16. Received 2018 June 29; in original form 2018 May 15

\begin{abstract}
We make the first attempt to find dwarf galaxies in eight Fermi-LAT extended, unassociated, source fields using Gaia DR2. After probing previously unexplored heliocentric distances of $d<20 \mathrm{kpc}$ with an extreme-deconvolution (XD) technique, we find no sign of a dwarf galaxy in any of these fields despite Gaia's excellent astrometric accuracy. Our detection limits are estimated by applying the XD method to mock data, obtaining a conservative limit on the stellar mass of $M_{*}<10^{4} \mathrm{M}_{\odot}$ for $d<20 \mathrm{kpc}$. Such a low stellar mass implies either a low-mass subhalo or a massive stripped-down subhalo. We use an analytic model for stripped subhalos to argue that, given the sizes and fluxes of the Fermi-LAT sources, we can reject the hypothesis that they owe to dark matter annihilation.
\end{abstract}

Key words: catalogues - astrometry - Galaxy: structure - Galaxy: halo - galaxies: dwarf - gamma-rays: general

\section{INTRODUCTION}

In the current $\Lambda$ CDM cosmological model, dark matter $(\mathrm{DM})$ is the dominant matter component of the Universe, comprising approximately $26.8 \%$ of its total mass-energy (e.g. Planck Collaboration et al. 2016). It is required to explain the rotation curves of stars and gas in galaxies (e.g. Rubin et al. 1980; Oh et al. 2011; Battaglia et al. 2013), strong and weak lensing of galaxies and clusters (e.g. Clowe et al. 2006; Harvey et al. 2015), and the growth of large scale structure (e.g. Springel et al. 2006; Baur et al. 2016), yet its nature remains elusive.

The latest observational evidence points to DM being a cold, collisionless, fluid (e.g. Clowe et al. 2006; Skordis et al. 2006; Dodelson 2011; Baur et al. 2016; Read et al. 2017), indicative of a new, fundamental, particle not present in the Standard Model of particle physics (e.g. Bertone et al. 2005). Of the many candidate particles, one of the most well-motivated is a Weakly Interacting Massive Particle

^ E-mail: ioana.ciuca.16@ucl.ac.uk
(WIMP), with a mass in the range $2 \lesssim m_{\chi} / \mathrm{GeV} \lesssim 2000 \mathrm{GeV}$ (e.g. Jungman et al. 1996). Depending on its mass and annihilation cross section, WIMP DM can annihilate or decay into Standard Model particles, including gamma rays (e.g. Silk \& Bloemen 1987). This theorized property of DM has prompted the search for gamma-ray signals from astronomical targets with high DM content as an indirect detection method, using data from space- and ground-based gammaray telescopes, such as the Large Area Telescope (LAT), aboard the Fermi satellite, which maps the whole sky from about $30 \mathrm{MeV}$ to $>500 \mathrm{GeV}$ (e.g. Atwood et al. 2009), and the planned CTA array (e.g. Acharya et al. 2013).

Dwarf spheroidal galaxies (dSphs) in the Milky Way represent a particularly clean target for gamma-ray emission due to DM annihilation because of their high mass-to-light ratios (e.g. Aaronson 1983; Pryor \& Kormendy 1990; Mateo 1998; Charbonnier et al. 2011; Bonnivard et al. 2015; Klop et al. 2017) and relatively low gamma-ray background emission from astrophysical sources (e.g. Lake 1990; Evans et al. 2004; Baltz et al. 2008; Winter et al. 2016). So far, the search for a DM gamma-ray annihilation signal from such systems 
has not yielded any significant detection in the frequency range probed by Fermi-LAT. However, it provides some of the most stringent constraints on the nature of DM particles in terms of their mass and annihilation cross-section (e.g. Albert et al. 2017; Calore et al. 2018). This is beginning to challenge the WIMP paradigm.

Usually, dSphs are identified in optical surveys and confirmed via deep photometric or spectroscopic follow-up (e.g. Belokurov et al. 2007). Then, a corresponding gamma-ray signal can be looked for in their direction (e.g. GeringerSameth et al. 2015; Albert et al. 2017; Calore et al. 2018). An alternative approach is to explore the association of stellar counterparts with "unassociated" gamma-ray sources, i.e. sources identified as such by Fermi-LAT but lacking counterparts at other wavelengths. The detection of a dSph at the position of a gamma-ray source would provide the first observational evidence of the association between a dSph and gamma-ray emission. If the gamma-ray source is spatially extended, then this would be a 'smoking gun' for DM annihilation (e.g. Bertoni et al. 2015, 2016; Calore et al. 2017).

Unassociated sources represent almost one third of all gamma-ray detected sources in the Third Fermi GammaRay Source Catalog (the 3FGL Acero et al. 2015). Among 3FGL unassociated sources, Bertoni et al. $(2015,2016)$ found that 3FGL J2212.5+0703 exhibits a spatially extended profile, with no other wavelength counterparts so far, and suggested that this could be a DM subhalo. Xia et al. (2017) found that the source 3FGL J1924.8-1034 also has a spatially extended profile at high significance, making it another possible DM subhalo candidate. We caution, however, that Fermi-LAT Collaboration \& Biteau (2018) did not find any evidence for an individual source with statistically significant extension at the position of these two objects; a model for two close point-like sources was preferred in both cases. Very recently, the Fermi-LAT Collaboration released the catalogue of extended high-latitude sources, $|b|>5^{\circ}$ (Fermi-LAT Collaboration \& Biteau 2018), where six newly unassociated extended objects are identified.

The European Space Agency's Gaia mission (Gaia Collaboration et al. 2016) has made the second data release of their unprecedented parallax and proper motion measurements for more than one billion stars brighter than $G<20$ mag (Gaia DR2; Gaia Collaboration et al. 2018; Lindegren et al. 2018). This provides us with a new window to find dSphs using proper motions in the inner Galactic halo, where the stellar density is too high to detect them from the photometric data alone (Antoja et al. 2015). In this paper, using both parallax and proper motion data from Gaia DR2, we search for dSphs in the fields of the two above mentioned Fermi-LAT sources as our primary target. Additionally, we apply the same technique to the six Fermi-LAT extended source fields recently found by Fermi-LAT Collaboration \& Biteau (2018).

We focus on the (heliocentric) distance range between 1 and $20 \mathrm{kpc}$. So far, no dSph galaxies have been found nearer than $20 \mathrm{kpc}$ from the Sun (Belokurov et al. 2014; Koposov et al. 2015; Drlica-Wagner et al. 2015; Bechtol et al. 2015; Kim et al. 2015; Kim \& Jerjen 2015; Laevens et al. 2015a,b; Luque et al. 2016). Only Draco II is estimated to be at a distance of $20 \mathrm{kpc}$ (Laevens et al. 2015b; Martin et al. 2016), but the vast majority ( $\gtrsim 70 \%$ ) of dSphs have been found at distances farther than $50 \mathrm{kpc}$. This owes to a combination of several effects. In the inner halo, dSph satellites are expected to be more diffuse both intrinsically and apparently: intrinsically because they are more prone to tidal disruption induced by the Galactic disc (e.g. D'Onghia et al. 2010; Garrison-Kimmel et al. 2017; Sawala et al. 2017); and apparently, because of the projected size which increases with decreasing distance. At larger apparent sizes, the contamination by field halo and disc stars plays an increasingly important role, washing out a dSph's signature on the sky. Thus, the inner halo within $20 \mathrm{kpc}$ from the Sun is an unexplored territory. Gaia DR2 can make a significant contribution over this distance range by making use of joint distance and kinematic information to find both phase space overdensities, and to separate dSph stars from the Milky Way foreground (Antoja et al. 2015). By combining our constraints on the presence or absence of dSphs at these small distances with the current lack of a DM annihilation signal from the more distant dSphs (Albert et al. 2017), we test the hypothesis that these unassociated Fermi-LAT sources owe to DM annihilation.

This paper is organised as follows. Section 2 describes the method to search for stellar counterparts to the FermiLAT unassociated, extended sources. In Section 3 we present the results of our search for a dSph within Gaia DR2. In Section 4, we discuss the detection limits of our method with Gaia DR2, and how the upper bound on $M_{*}$ translates into bounds on the likely pre-infall halo mass of a dSph. In Section 5, we use our detection limits from Section 4 to determine whether or not the Fermi-LAT sources could be explained by DM annihilation from a nearby subhalo. We find that the sizes and fluxes are inconsistent with the DM subhalo hypothesis. Finally, a summary and discussion of our results are presented in Section 6.

\section{DATA AND METHOD}

We selected a region of the sky within $2^{\circ}$ and $1^{\circ}$ from our two primary targets of Fermi-LAT unassociated gamma-ray sources: 3FGL J2212.5+0703 $\left(l=68.74, b=-38^{\circ} .57\right)$ and 3 FGL J1924.8+1034 $\left(l=27^{\circ} .16, b=-12^{\circ} .17\right)$, respectively. We also applied the same technique within $1^{\circ}$ to the six extended unassociated gamma-ray sources in Fermi-LAT Collaboration \& Biteau (2018), namely FHES J1501.0-6310 $(l=316.95, b=-3.89)$, FHES J1723.5-0501 $(l=17.90$, $\left.b=16^{\circ} .96\right)$, FHES J1741.6-3917 $(l=350.73,-4.72)$, FHES $\mathrm{J} 2129.9+5833\left(l=99^{\circ} .13, b=5.33\right)$, FHES J2208.4+6443 $\left(l=106^{\circ} .62, b=7^{\circ} .15\right)$ and FHES J2304.0+5406 $\left(l=107^{\circ} .50\right.$, $b=-5.52)$.

We downloaded all of the Gaia DR 2 stars in these regions from the Gaia archive site (https://gea.esac.esa. int/archive/) and Centre de Données astronomiques de Strasbourg (CDS). We employed two different strategies for finding a dSph galaxy depending on whether it is located at a distance farther than $d=10 \mathrm{kpc}$ or not.

We used both spatial and proper motion information from Gaia DR2 to determine whether we can observe the stellar imprint of a dSph galaxy at the position of each Fermi-LAT unassociated, extended, gamma-ray source. For this purpose, we selected the stars around a radius of $2^{\circ}$ and $1^{\circ}$ centred on the position of 3FGL J2212.5+0703 and 
3FGL J1924.8+1034, respectively. The smaller field of view for 3FGL J1924.8+1034 was chosen to reduce the contamination from the field stars at low Galactic latitude. The additional 6 fields in Fermi-LAT Collaboration \& Biteau (2018) are also low Galactic latitude fields, and therefore we chose $1^{\circ}$ field radius for these sources as well.

When searching for a dSph galaxy farther than $d=$ $10 \mathrm{kpc}$ away, we applied the same parallax filter used in Antoja et al. (2015), namely, we discard stars for which $\varpi-\sigma_{\varpi}>0.1$ mas, where $\varpi$ represents the parallax and $\sigma_{\varpi}$ is the parallax uncertainty. This filter corresponds to eliminating stars located at a distance of less than $10 \mathrm{kpc}$ within parallax uncertainties and aims to minimize contamination from foreground stars. After these quality cuts, we are left with 17,747 and 125,891 stars in the fields of 3FGL J2212.5+0703 and 3FGL J1924.8+1034, respectively. For the additional 6 fields, we obtain $467,082,61,091,670,424,120,156,82,638$ and 115,848 stars in the fields of FHES J1501.0-6310, FHES J1723.5-0501, FHES J1741.6-3917, FHES J2129.9+5833, FHES J2208.4+6443 and FHES J2304.0+5406, respectively.

We then used an Extreme-Deconvolution (XD, Bovy et al. 2011) Gaussian Mixture Model (XDGMM, Holoien et al. 2017) to perform density estimation on a fourdimensional dataset comprised of the stellar position in Galactic longitude and latitude, and the RA and DEC proper motion measurements. Our aim is to detect a group of stars with similar proper motions which are also concentrated on the sky. As XD has been proven to be a powerful tool in estimating the noise-free underlying distributions of astrophysically relevant quantities (Hogg et al. 2005; Bovy et al. 2009, 2012), we have decided to use it for the current purpose. XD allows us to assume Gaussian errors in the Gaia DR2 proper motions and parallaxes (Hogg et al. 2005; Bovy et al. 2009, 2012) and also to take into account the correlation between the measurement of RA and DEC proper motions. We do not take into account the measurement uncertainties in the stellar position or correlations between the positions and proper motions, because the uncertainties in the position in the Gaia DR2 are very small. For this analysis, we applied a small constant uncertainty of 0.01 in the stellar positions for practical reasons, whose effect is small enough not to affect our results.

Fig. 1 shows an example of the XD method applied to a dSph galaxy of stellar mass of $M_{*}=5000 \mathrm{M}_{\odot}$, halfmass radius of $r_{\mathrm{h}}=50 \mathrm{pc}$, and moving at a velocity of $V_{\text {gal }}=50 \mathrm{~km} \mathrm{~s}^{-1}$ at a distance of $d=10 \mathrm{kpc}$ in the search field of 3FGL J2212.5+0703. We constructed the background stars using Galaxia (Sharma et al. 2011) in the field of 3FGL J2212.5+0703. The stellar catalogue, representative of a dSph galaxy, was made with SNAPDRAGONS (Hunt et al. 2015) assuming an age of 12 Gyr, a metallicity of $Z / Z_{\odot}=0.01$, and a Salpeter initial mass function. The dSph galaxy's dynamical properties were modelled following a simple Plummer distribution with $r_{\mathrm{h}}=50 \mathrm{pc}$, and an isotropic velocity distribution with a dispersion of $\sigma=10 \mathrm{~km} / \mathrm{s}$. We applied the expected Gaia DR2 uncertainties at https://www.cosmos.esa.int/web/gaia/dr2 to the data for both the dSph galaxy and field stars. In Fig. 1, we see that XD can find the modelled dSph galaxy in proper motion space. We found that an excessive number of components in the XD does not penalise the detection of the dSph (see also Anderson et al. 2017). However, it may result
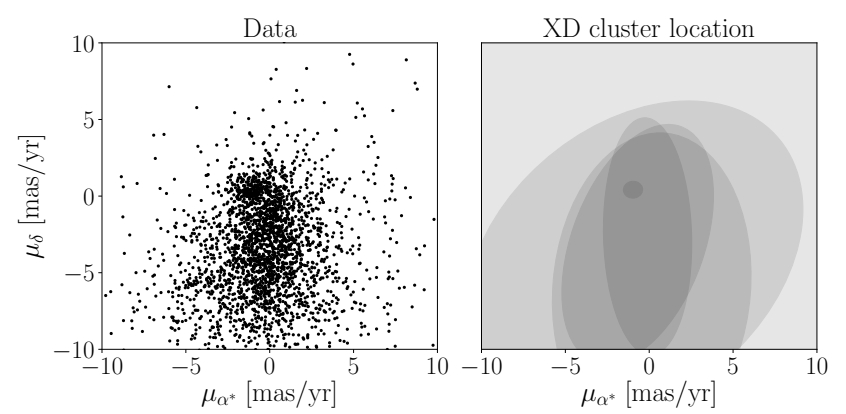

Figure 1. Example of a clear detection of a cluster associated with a dSph galaxy obtained by applying the XD method to mock data. We assumed a dSph galaxy with a stellar mass of $\boldsymbol{M}_{*}=5000 \mathrm{M}_{\odot}$, a half-mass radius of $r_{\mathrm{h}}=50 \mathrm{pc}$, and moving at a velocity of $V_{\mathrm{gal}}=50 \mathrm{~km} \mathrm{~s}^{-1}$ at a distance of $d=10 \mathrm{kpc}$ in the search field of 3FGL J2212.5+0703. We used $N=6$ components in the XD. The left panel shows the the input stellar distribution in proper motion space, compressed by a factor of 5 . The right panel shows the Gaussian components found by XD, including the cluster associated with the mock dSph galaxy centered at a

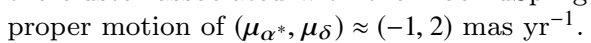

in overfitting the data. We find that using $K=25$ components is a good model for most of the fields. However, for the low Galactic latitude fields, we additionally used $K=50$ for the fields of 3FGL J1924.8+1034, FHES J1501.0-6310 and FHES J2304.0+5406 and $K=100$ for the field of FHES J1741.6-3917. The numbers of components for each field are determined such as to recover a dSph with our conservative stellar mass limit, as discussed in Section 4.

For the search for a dSph galaxy located at distances $d<$ $10 \mathrm{kpc}$, we employed a different parallax cut. The parallax cut is dependent on distance, $d_{\text {in }}$, as: $1 /\left(2 d_{\text {in }}\right)<\varpi<1.0 / d_{\text {in }}$ and changes as we are probing a distance range between 1.0 and $10 \mathrm{kpc}$ in increments of $d_{\text {in }}$ of $1 \mathrm{kpc}$ up to $5 \mathrm{kpc}$, and then $2 \mathrm{kpc}$ up to $9 \mathrm{kpc}$. In addition, we applied a brighter magnitude cut for 17,18 and $19 \mathrm{mag}$ for $d_{\text {in }}=1,2$ and $3 \mathrm{kpc}$ cases, respectively, to use only the stars with better parallax accuracy. A dSph in this distance range, whether completely disrupted or not, will likely only be recovered as a moving group with no discernible spatial overdensity, due to its large angular extent. However, we found that this does not penalise the XD search for a dSph. Hence, XD was applied to both proper motion and a spatial distribution as described above.

\section{GAIA DR2 SEARCH FOR A DWARF GALAXY}

We applied XD to the samples of stars with $d<10 \mathrm{kpc}$ and $d>10 \mathrm{kpc}$, filtered as mentioned above, in the fields of 3FGL J2212.5 + 0703, 3FGL J1924.8 + 1034, FHES J1501.06310, FHES J1723.50501, FHES J1741.63917, FHES J2129.9 + 5833, FHES J2208.4 + 6443 and FHES J2304.0 + 5406. The intensive XD search undertaken after a careful visual inspection of all the fields using TOPCAT (Taylor 2005) yielded no evidence for a dSph in any of these fields. In the next section, we provide our conservative detection limits for each field. In 

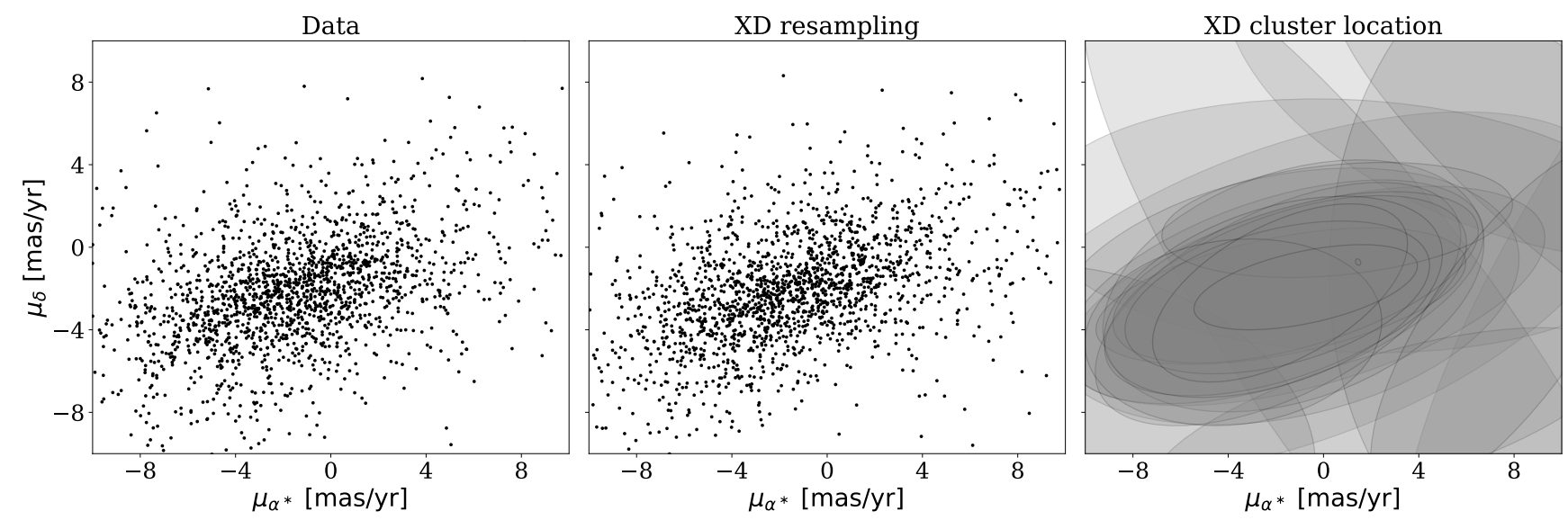

Figure 2. A detection of an open cluster - NGC 7438 - in the field of FHES J2304.0+5406. The left panel shows the data after filtering with $d_{\text {in }}=1 \mathrm{kpc}$, i.e. $0.5<\varpi(\mathrm{mas})<1$. The right panel shows the detected Gaussian models with the XD method. The middle panel presents the distribution sampled from the detected models. The open cluster is marked by a small circle around $\left(\mu_{\alpha^{*}}, \mu_{\delta}\right)=$

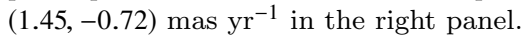

this section, we provide examples of some interesting cases that demonstrate the validity of our approach.

While we found no evidence for new dSphs in these Fermi-LAT fields, we did find overdensities of stars associated with known star clusters, demonstrating that the XD method can successfully find phase space structures in the Gaia DR2 data. Fig. 2 shows that XD can be used to successfully detect faint stars in the outskirts of NGC 7438 ( $l=106.7, b=-4.90)$ which is at a distance of $d=1 \mathrm{kpc}$ (Dias et al. 2002). Our detected proper motion is $\left(\mu_{\alpha^{*}}, \mu_{\delta}\right)=$ $(1.45,-0.72)$ mas $\mathrm{yr}^{-1}$.

We also noticed that there is part of a globular cluster, NGC 6366, in the field of FHES J1723.5-0501. These stars are also clearly identified with the $\mathrm{XD}$, and we found

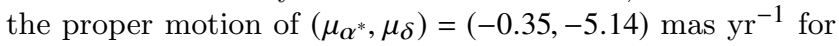
NGC 6366. The XD identified Ruprecht $112(d=1.76 \mathrm{kpc})$ in the filed of FHES J1501.0-6310, when we set $d_{\text {in }}=2 \mathrm{kpc}$ and also $d_{\text {in }}=3 \mathrm{kpc}$. Because fainter stars with lower parallax accuracy tend to be found at a higher distance (e.g. Luri et al. 2018), our method detects the stellar system when we use a larger $d_{\text {in }}$ than the distance of the stellar system.

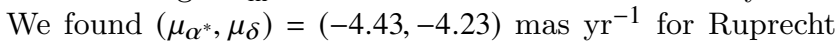
112. Finally, Trumpler $29(d=0.76 \mathrm{kpc})$ is identified in the FHES J1741.6-43917 field. We obtain a proper motion of $\left(\mu_{\alpha^{*}}, \mu_{\delta}\right)=(0.49,-2.30) \mathrm{mas}_{\mathrm{yr}^{-1}}$ for Trumpler 29 . All the above distances to the open clusters are from the catalogue of Dias et al. (2002). Note that our proper motions identified with the XD are different from those in Dias et al. (2002). This is likely due to the improved astrometric accuracy of the Gaia data; we will explore this further in future work.

\section{MOCK DATA ANALYSIS: DETECTION LIMITS FOR DWARFS IN GAIA DR2}

In this section, we estimate the detection limits for the $\mathrm{XD}$ method applied to the Gaia DR2 data. We first estimate our detection limit using mock data as described in Section 2 for one of our primary Fermi-LAT fields, 3FGL J2212.5+0703. We then place the mock dSph model with the estimated upper limit into the Gaia data of each field and confirm that the mock dSph can be recovered in all of our fields up to a distance of $d=20 \mathrm{kpc}$.

To estimate the detection limit in the 3FGL J2212.5+0703 field, we set up mock data as described in Section 2. We placed a mock dSph with $M_{*}=10^{3}-10^{4} \mathrm{M}_{\odot}$, $r_{h}=20-100 \mathrm{pc}$ and $V_{\mathrm{gal}}=20-100 \mathrm{~km} \mathrm{~s}^{-1}$ at a distance of $d=1-30 \mathrm{kpc}$. As in Section 2, the field-star catalogues were made with Galaxia, applying Gaia DR2 uncertainties. Note that our goal is to place a conservative upper limit on the detection of a dSph in Gaia DR2. The detection limits are sensitive to the parameters mentioned above, and precise evaluation would require more sophisticated mock data and statistical analyses that are beyond the scope of this paper. As we shall show, however, the conservative limit is sufficient for our calculation of the implications of our results for DM annihilation in Section 5 .

We found that $r_{h}$ is not a critical parameter for the detection limit, while our detection is sensitive to $V_{\text {gal }}$. Hence, in the panels of Fig. 3 we provide our detection limit parameter survey results for three different $V_{\text {gal }}$ for the 3FGL J2212.5+0703 field. In all cases, the XD method reliably recovers a dSph of $M_{*}>10^{4} \mathrm{M}_{\odot}$ at $d<20 \mathrm{kpc}$.

Based on this result, we now derive a conservative detection threshold for our other fields. For this, we set up mock dSphs with $M_{*}=10^{4} \mathrm{M}_{\odot}, r_{h}=50 \mathrm{pc}, V_{\mathrm{gal}}=$ $20 \mathrm{~km} \mathrm{~s}^{-1}$ and $d=1-20 \mathrm{kpc}$ for each field, as above, taking into account the different dust extinction in each field. (For this we use the SNAPDRAGONS code that uses the same extinction model as Galaxia.) For the fields 3FGL J2212.5+0703, FHES J1723.5-0501, FHES J2129.9+5833 and FHES J2208.4+6443, the XD method with $K=$ 25 components recovered the mock dSph up to $20 \mathrm{kpc}$. In the 3FGL J1924.8+1034, FHES J1501.0-6310 and FHES $\mathrm{J} 2304.0+5406$ fields, the dSph at $d=15$ and $20 \mathrm{kpc}$ required us to use $K=50$ components. In the densest field, FHES J1741.6-3917, the mock dSphs at $d>10 \mathrm{kpc}$ required $K=100$ components to be detected. In our search for dSphs in Section 3, we used the number of components, $K$, as de- 

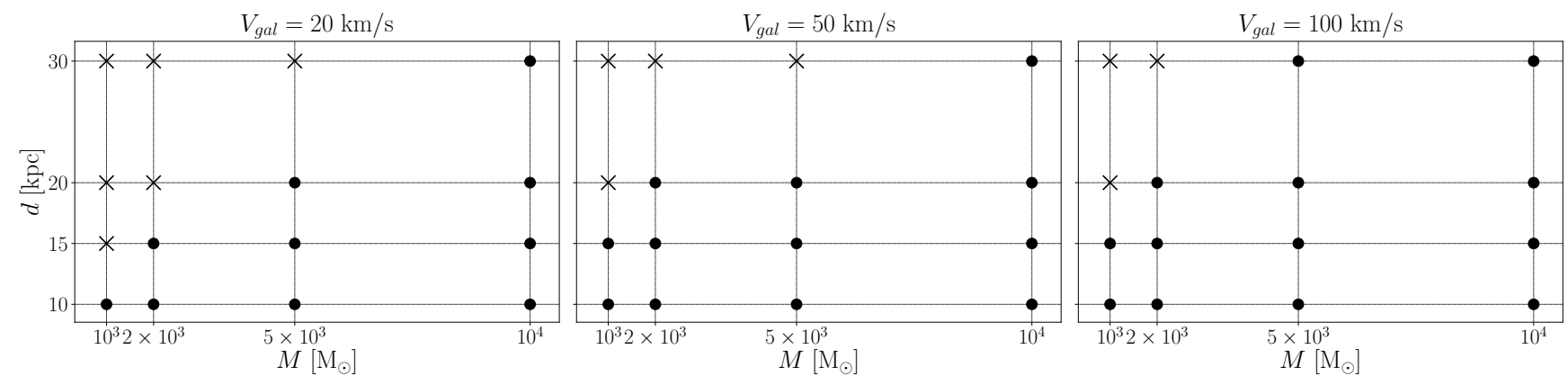

Figure 3. The detectability of a mock dSph galaxy as a function of its mass, distance and velocity using the XD method applied to the 3FGL J2212.5+0703 field. The left, middle and right panels show results for a velocity of 20, 50 and $100 \mathrm{~km} \mathrm{~s}^{-1}$, respectively. Solid circles (crosses) represent the parameters for which a dSph can (cannot) be found in the mock data with the XD.

termined from these mock data tests, to search for dSphs in each field. From the above analysis, we place a conservative upper limit on our detection threshold of $M_{*}>10^{4} \mathrm{M}_{\odot}$ for any dSph galaxy along the line of sight to our sample of unassociated, extended, Fermi-LAT within $d=20 \mathrm{kpc}$.

\section{DARK MATTER INTERPRETATION OF THE Fermi-LAT UNASSOCIATED, EXTENDED SOURCES}

It was pointed out that the gamma-ray signatures from our primary two Fermi-LAT unassociated sources 3FGL J2212.5+0703 and 3FGL J1924+1034 were consistent with DM annihilation into a $b \bar{b}$ final state with masses around tens of GeV (Bertoni et al. 2016; Xia et al. 2017). Since the mass of and distances to these objects were unknown, degeneracies between them and the annihilation cross section remained.

For 3FGL J2212.5+0703, the radius that contains $68 \%$ of the total gamma-ray photons is found to be $\sigma=0.25$ (Bertoni et al. 2016); for 3FGL J1924+1034 it is $\sigma=0.15$. If the density profile of the DM subhalo is well approximated by the Navarro-Frenk-White profile, characterized by the scale radius $r_{s}$ and characteristic density $\rho_{s}$ (Navarro et al. 1997), $\sim 70 \%$ of the total annihilation happens within $0.5 r_{s}$. Therefore, we assume $\theta_{s} \equiv r_{s} / d \simeq 0.5\left(0^{\circ} 3\right)$ for $3 \mathrm{FGL}$ $\mathrm{J} 2212.5+0703$ (3FGL J1924+1034). This is based on the assumption that the tidal stripping is not too strong such that the tidal truncation radius is still larger than the scale radius, $r_{t}>r_{s}$, which is shown to be the case below.

To constrain the possibility that the Fermi-LAT sources we study in this paper owe to DM annihilation, we need to obtain a robust upper bound on the pre-infall halo mass, $M_{200}$, of dSphs with $M_{*}<10^{4} \mathrm{M}_{\odot}$ and $d<20 \mathrm{kpc}$ that lie below our detection threshold. This is because, at a fixed observed gamma-ray flux, the most massive, dense, halos minimise the inferred annihilation cross section. This then maximises the chance of consistency with the null detection from the known dSphs with $d>20 \mathrm{kpc}$.

Unfortunately, a satellite galaxy with $M_{*}<10^{4} \mathrm{M}_{\odot}$ is likely to have lost any tight relation between $M_{*}$ and its pre-infall $M_{200}$ (Gatto et al. 2013; Ural et al. 2015; Read et al. 2017). However, we can estimate an upper bound on $M_{200}$ by comparison with the surviving 'classical' dSphs, and via cosmological simulations of Milky Way-mass halos (e.g. Diemand et al. 2007). For the former, estimates of $M_{200}$ for classical dSphs like Draco and Ursa Minor with purely old stellar populations lie in the range $M_{200}=(2-5) \times 10^{9} \mathrm{M}_{\odot}$ (Read \& Erkal 2018, in prep.). However, to be consistent with our detection threshold, these 'classical' dSphs would have to lose over $90 \%$ of their stellar mass (McConnachie 2012). The tidal stripping and shocking required to achieve this would lower their central dark matter densities by up to a factor of $\sim 10$ (e.g. Read et al. 2006), significantly reducing the gamma-ray flux from DM annihilation. Indeed, a search for surviving subhalos in the Via Lactea II simulation (Diemand et al. 2007) yielded only one subhalo with a pre-infall mass $M_{200}>10^{9} \mathrm{M}_{\odot}$ inside $d<20 \mathrm{kpc}$, a subhalo with $M_{200}=1.1 \times 10^{9} \mathrm{M}_{\odot}$. Given the above arguments, we place a conservative upper bound on the pre-infall halo mass of $M_{200}<10^{9} \mathrm{M}_{\odot}$ for surviving dSphs with $M_{*}<10^{4} \mathrm{M}_{\odot}$ and $d<20 \mathrm{kpc}$. We consider this to be a strict upper bound since it does not account for subhalo depletion by the Milky Way disc (e.g. D'Onghia et al. 2010).

Armed with an upper bound $M_{200}$, we now consider the effects of tidal mass loss due to the orbit of this subhalo around the Milky Way. We model this by using the analytic prescription in Bartels \& Ando (2015) and Hiroshima et al. (2018), assuming a $10^{12} M_{\odot}$ host halo for the Milky Way.

We first consider pre-infall subhalo masses of $(0.8$ 1.2) $\times 10^{7} M_{\odot}$ in order to model an example case, which is well below the upper limit from our non-detection of a dSph, but is chosen to illustrate our methodology. For a reference value of distance, we adopt $d=10 \mathrm{kpc}$. In Fig. 4 , we show the probability density function (PDF) of the subhalo mass after tidal stripping, $P(m)$ (top left), and of the ratio of tidal truncation radius and the scale radius, $r_{t} / r_{s}$ (top right). Although the subhalo can lose significant fraction of its mass due to the tidal effect, its truncation radius $r_{t}$ is kept larger than the scale radius $r_{s}$. This is because we also take into account evolution of $r_{s}$ and $\rho_{s}$ (Peñarrubia et al. 2010), which compensates that of $r_{t}$. In the bottom left panel of Fig. 4, we show PDF of the angle size corresponding to the scale radius, $P\left(\theta_{s}\right)$ (middle). We see that for this case, the gammaray source extension of $\sigma=0.25$ (3FGL J2212.5+0703; vertical red line) can be consistent with a pre-infall mass of $M_{200}=10^{7} M_{\odot}$.

The gamma-ray flux from DM annihilation (assuming 

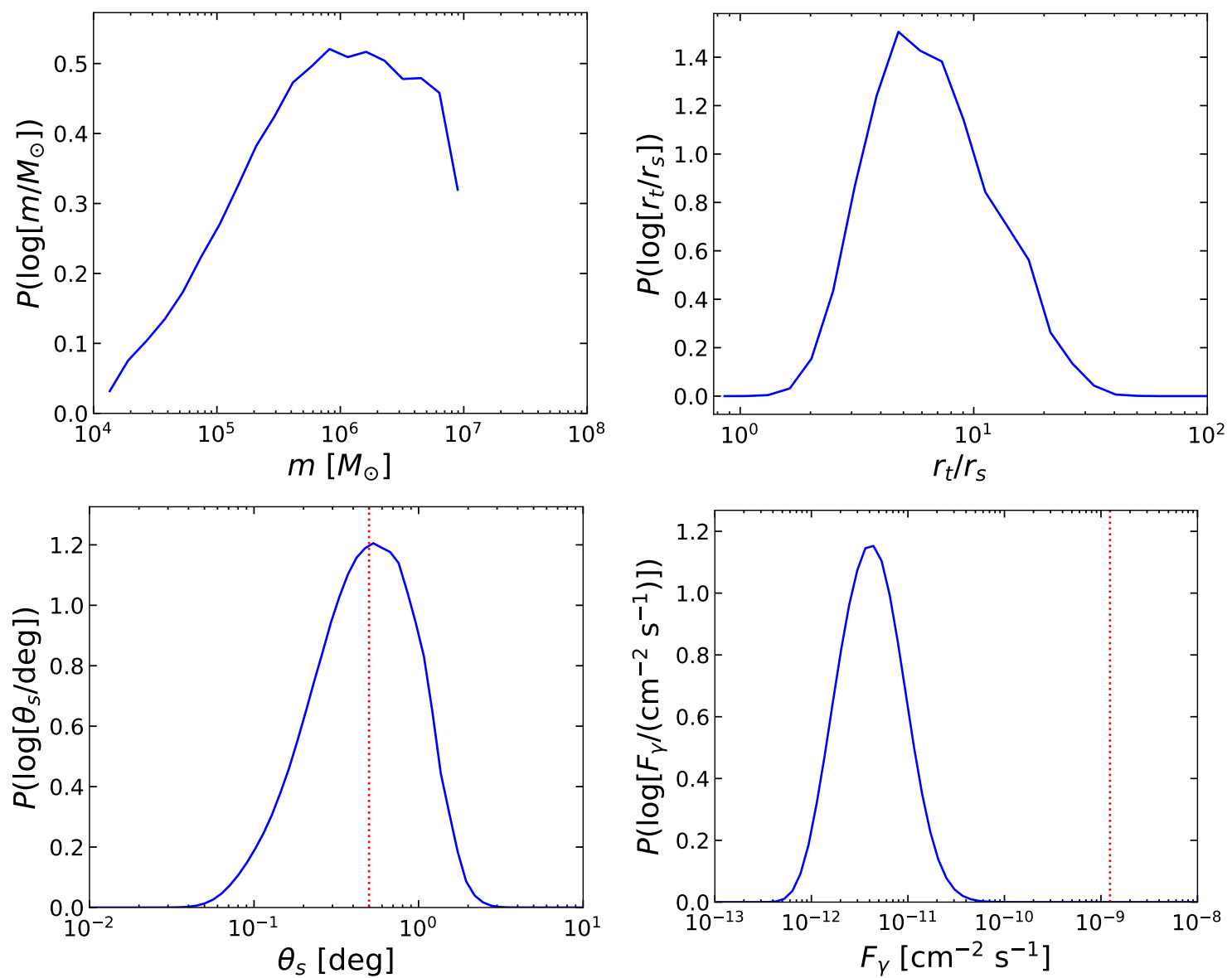

Figure 4. PDFs of post-infall subhalo mass (top left), ratio of tidal truncation radius and scale radius, $r_{t} / r_{s}$ (top right), the angle corresponding to the scale radius of the subhalo (bottom left), and the gamma-ray flux, $F_{\gamma}$, assuming $m_{\mathcal{X}}=25 \mathrm{GeV}$ and $\langle\sigma v\rangle=$ $2 \times 10^{-26} \mathrm{~cm}^{3} \mathrm{~s}^{-1}$ (bottom right). In this example, the pre-infall halo mass is $M_{200}=10^{7} M_{\odot}$ and the distance to the subhalo is $d=10 \mathrm{kpc}$. The scale radius and flux inferred from the Fermi-LAT gamma-ray distribution for 3 FGL J2212.5+0703 $\left(\theta_{s}=0.5\right)$ are shown as vertical red dotted lines on the middle and bottom panels for comparison.

that DM is made of Majorana fermions) is calculated as:

$F_{\gamma}=\frac{\langle\sigma v\rangle N_{\gamma, \text { ann }}}{2 m_{\chi}^{2}} \frac{1}{4 \pi d^{2}} \int d V \rho_{\chi}^{2}(r)$

where $m_{\chi}$ is the DM particle mass, $\langle\sigma v\rangle$ is the annihilation cross section, $N_{\gamma, \text { ann }}$ is the number of gamma-ray photons emitted per annihilation, and $\rho_{\mathcal{X}}(r)$ is the DM density profile of the subhalo. ${ }^{1}$ For a representative calculation, we adopt $m_{\chi}=25 \mathrm{GeV},\langle\sigma v\rangle=2 \times 10^{-26} \mathrm{~cm}^{3} \mathrm{~s}^{-1}$ (e.g., Steigman et al. $2012)$, and $N_{\gamma \text {, ann }}=3.7$ that is for $E_{\gamma}>1 \mathrm{GeV}$ in the case of annihilation into a $b \bar{b}$ final state. The bottom panel of Fig. 4 shows the PDF of the gamma-ray flux $F_{\gamma}$ corresponding to this example case of $M_{200}=10^{7} M_{\odot}$ and $d=10 \mathrm{kpc}$. It shows

1 Strictly speaking, Eq. (1) is valid only if the source extension is small, $\theta_{s} \ll 1$. For smaller distances or larger masses, one has to implement the line-of-sight integral to compute the gammaray intensity and integrate it over the solid angle to obtain the flux. However, the observed extensions of the Fermi unassociated sources are at most $O(1)^{\circ}$, and hence, in order to explain the observed features in terms of DM annihilation, using Eq. (1) instead of the more accurate line-of-sight integral is well justified. that, for this case in which one can explain the source extension well, the mean gamma-ray flux above $1 \mathrm{GeV}$ will be $4 \times 10^{-12} \mathrm{~cm}^{-2} \mathrm{~s}^{-1}$, which is smaller than the observed flux of $1.24 \times 10^{-9} \mathrm{~cm}^{-2} \mathrm{~s}^{-1}$ for 3FGL J2212.5+0703 (Bertoni et al. 2016) by more than two orders of magnitude. This means that in order to explain the gamma-ray signal from 3FGL J2212.5+0703 in terms of DM annihilation, with a subhalo of this mass and distance, the annihilation cross section needs to be larger by more than two orders of magnitude than the canonical value $\left(\langle\sigma v\rangle=2 \times 10^{-26} \mathrm{~cm}^{3} \mathrm{~s}^{-1}\right)$ adopted here as well as in Bertoni et al. (2016). Such a large cross section has already been excluded from the analysis of the known dSphs in the Milky Way (Albert et al. 2017) at high significance, even when systematic uncertainties are taken into account (Calore et al. 2018). This illustrates how we can combine our Gaia DR2 dSph detection limits with DM annihilation constraints from the known Milky Way dSphs to constrain or rule out a DM annihilation interpretation of the unassociated Fermi-LAT sources.

In Fig. 5, we show the upper limits on the angular extension $\theta_{s}$ (top panel) and on the gamma-ray flux (bottom panel) as a function of the dSph distance for the subhalo masses of $10^{6}, 10^{7}, 10^{8}$ and $10^{9} M_{\odot}$ ( solid blue lines, 

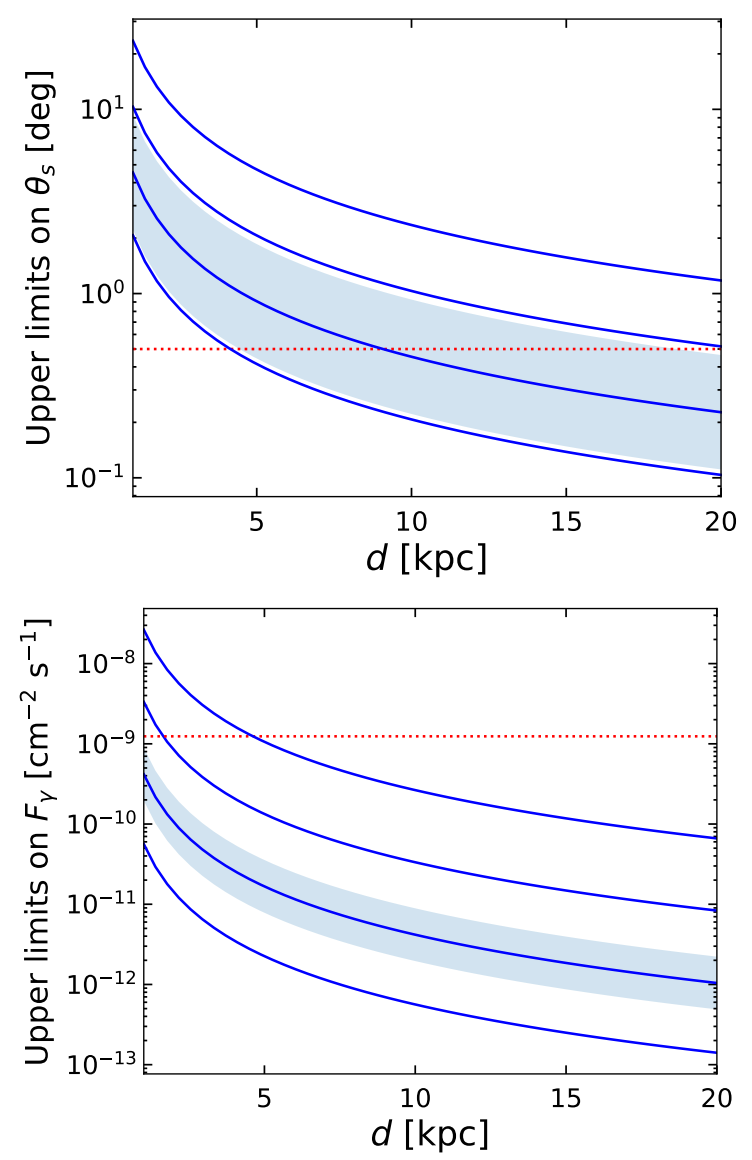

Figure 5. Upper limits on the angular size corresponding to the scale radius $\theta_{s}$ (top) and gamma-ray flux (bottom) from DM annihilation $F_{\gamma}$ as a function of distance $d$. From top to bottom, the blue solid lines correspond to pre-infall halo masses: $\boldsymbol{M}_{200}=$ $10^{9}, 10^{8}$, and $10^{6} M_{\odot}$, respectively. For the $M_{200}=10^{7} \mathrm{M}_{\odot}$ subhalo, we show the $1 \sigma$ scatter in our theoretical modelling as a light blue band. This scatter owes primarily to the uncertain tidal mass loss history of the subhalo. The horizontal red dotted lines show the measured values from the Fermi-LATdata analysis for 3FGL $\mathrm{J} 2212.5+0703$.

from bottom to top, respectively). (Recall that the upper limit on pre-infall mass is $M_{200}=10^{9} M_{\odot}$.) For the case of $M_{200}=10^{7} M_{\odot}$, we also show the $1 \sigma$ uncertainty in the modelling that owes primarily to the uncertain history of tidal mass stripping (blue band).

We note that we also checked these results using a simple approach that does not account for tidal stripping and is based on the concentration parameterisation from Moliné et al. (2017). We find a good agreement between the two different approaches within our quoted uncertainties. The differences come from the fact that small changes of internal profiles such as $r_{s}$ and $\rho_{s}$ (e.g., Peñarrubia et al. 2010) have been taken into account for the former approach based on Bartels \& Ando (2015) and Hiroshima et al. (2018), but not in the latter.

Fig. 6 shows the $1 \sigma$ and $2 \sigma$ contours in the plane, $\left(\theta_{s}, F_{\gamma}\right)$, for pre-infall masses $M_{200}=10^{7} M_{\odot}$ and $10^{9} M_{\odot}$ and distances $d=3 \mathrm{kpc}$ and $10 \mathrm{kpc}$. For the cases of 3FGL J2212.5+0703 (star; Bertoni et al. 2016) and 3FGL J1924.8-

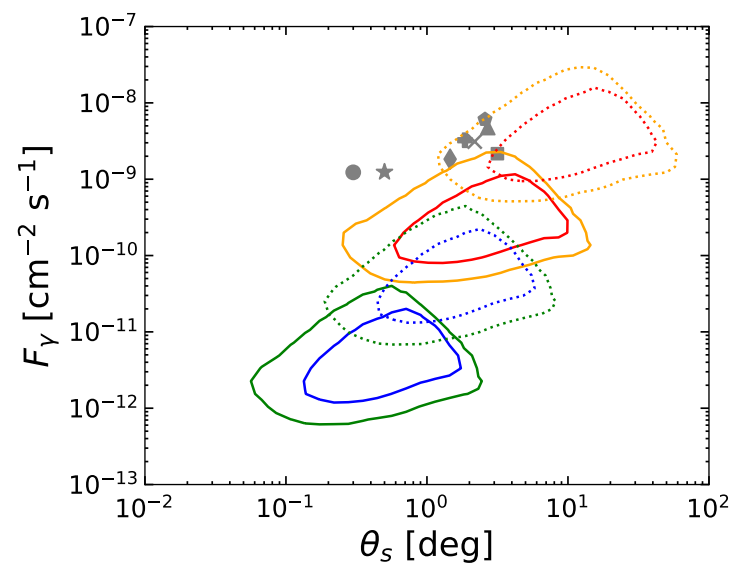

Figure 6. $1 \sigma$ and $2 \sigma$ regions of the joint $\mathrm{PDF}, P\left(\theta_{s}, F_{\gamma}\right)$, for subhalos with a pre-infall mass of $M_{200}=10^{7} M_{\odot}$ at $d=10 \mathrm{kpc}$ (lower solid), $10^{9} M_{\odot}$ at $10 \mathrm{kpc}$ (upper solid), $10^{7} M_{\odot}$ at $3 \mathrm{kpc}$ (lower dotted), and $10^{9} M_{\odot}$ at $3 \mathrm{kpc}$ (upper dotted). Measured values for the eight Fermi unassociated sources are shown for comparison: 3FGL J2212.5+0703 (star), 3FGL J1924.8-1034 (circle), FHES J1501.0-6310 (pentagon), FHES J1723.5-0501 (diamond), FHES J1741.6-3917 (square), FHES J2129.9+5833 (cross), FHES J2208.4+6443 (plus), and FHES J2304.0+5406 (square).

1034 (circle; Xia et al. 2017), the measured signals are incompatible with a DM annihilation interpretation, even for halos at the pre-infall halo mass upper bound of $M_{200}=10^{9} \mathrm{M}_{\odot}$. We note that recently Fermi-LAT Collaboration \& Biteau (2018) reported that the gamma-ray signals from both 3FGL J2212.5+0703 and 3FGL J1924.8-1034 are better explained by two point sources rather than a single extended source. Our results here are consistent with this finding, excluding the hypothesis of DM annihilation from a subhalo within $d<20 \mathrm{kpc}$.

Fermi-LAT Collaboration \& Biteau (2018) found six additional unassociated extended sources. In this work, we analysed all of them and found no signature of a dSph galaxy in any of these fields. Among them, FHES J1723.5-0501, FHES J1741.6-3917, and FHES J2304.0+5406 were found to have an energy spectrum harder than $E^{-2}$, as is expected from DM annihilation. In Fig. 6 we also show measured values of $\left(\theta_{s}, F_{\gamma}\right)$ for these six Fermi-LAT unassociated sources. As can be seen, none of these unassociated sources is compatible with DM annihilation within our $2 \sigma$ contours, unless the pre-infall mass of the subhalo is close to the upper limit, $10^{9} M_{\odot}$, and it is located around $d=3 \mathrm{kpc}$. Such a massive pre-infall halo is very unlikely to be found so close to the Sun - especially when accounting for subhalo depletion by the disc (see the discussion on this, above). Since the annihilation cross section $\langle\sigma v\rangle=2 \times 10^{-26} \mathrm{~cm}^{3} \mathrm{~s}^{-1}$ adopted here is already in tension with other data analyses (e.g., Albert et al. 2017) for $25 \mathrm{GeV}$ WIMPs, possibilities with lighter $\left(\lesssim 10^{9} M_{\odot}\right)$ halos are excluded.

Finally, we discuss a few caveats, all of which we believe make our conclusion, above, stronger. Firstly, the discussions up to this point have been based on the assumption that the subhalo only experienced tidal stripping due to gravitational potential of the spherical host halo. However, subhalos orbiting within $d<20 \mathrm{kpc}$ are likely on eccentric orbits and will additionally experience tidal shocks that can 
lower their central density by up to a factor of $\sim 10$ (e.g. Read et al. 2006). Furthermore, we have not taken into account the effect of the Milky Way disc that depletes the number of substructures within $20-30 \mathrm{kpc}$ by a factor of $\sim 2$ (D'Onghia et al. 2010). Including such effects will lower the gamma-ray flux for a given pre-infall $M_{200}$ requiring us to further increase the annihilation cross section, further exasperating the tension with the constraints from the known Milky Way dSphs. Finally, we have only considered subhalos within $d<20 \mathrm{kpc}$. Lighter subhalos at larger distances than this would be too faint to be consistent with the observed fluxes unless they have large annihilation cross sections at odds with the constraints from the known dSphs in the Milky Way. Massive subhalos with $M_{200}>10^{9} \mathrm{M}_{\odot}$ at larger distances would have a readily detectable stellar counterpart, and then a DM annihilation signal from the known dSphs in the Milky Way should be already detected (see the discussion, above). Hence, this can also be excluded. We conclude that none of the unassociated, extended, Fermi-LAT sources studied here can have a DM annihilation origin.

\section{SUMMARY}

Using the new second data release of the Gaia mission, we have made the first attempt to find a dSph towards eight Fermi-LAT extended unassociated source fields. Our goal was to link the gamma-ray emission, already detected by Fermi-LAT, to a possible optical counterpart within the framework of annihilating DM. Gaia's superb astrometric accuracy provides a new window for searching for dSphs in the inner Galactic halo $(d<20 \mathrm{kpc})$ based on the proper motion and parallax of stars (Antoja et al. 2015). We applied an advanced statistical method, the Extreme-Deconvolution (XD, Bovy et al. 2011) Gaussian Mixture Model (XDGMM, Holoien et al. 2017), to properly take into account the uncertainties and correlations in proper motion space. Unfortunately, we found no detection indicative of a signature of a dSph galaxy in any of these fields placed within the Gaia data. We then estimated the detection limits for a dSph galaxy by applying XD to mock data. We obtained a conservative limit on the stellar mass of any undetected dSph of $M_{*}<10^{4} \mathrm{M}_{\odot}$ for $d<20 \mathrm{kpc}$. We showed that this corresponds to an upper limit on the pre-infall halo mass of $M_{200}<10^{9} \mathrm{M}_{\odot}$.

Using an analytical model of subhalo mass stripping that has been calibrated against numerical simulation results, combined with current limits on the DM annihilation cross section from known Milky Way dSphs, we estimated the gamma-ray flux and source size as a function of pre-infall halo mass for all eight Fermi-LAT sources. We concluded that our model rejects the possibility of a DM annihilation scenario for the two sources: 3FGL J2212.5+0703 (Bertoni et al. 2016) and 3FGL J1924.8-1034 (Xia et al. 2017) if the pre-infall DM halo mass is less than $M_{200}<10^{9} \mathrm{M}_{\odot}$ at distance of $d<20 \mathrm{kpc}$, as constrained by our work. If the subhalo is farther away than $20 \mathrm{kpc}$, then its DM halo has to be larger than $M_{200}=10^{9} \mathrm{M}_{\odot}$. Such a dSph would have a clear stellar counterpart that should have been detected in the existing photometric data in these fields. Furthermore, if there were a dSph at $d>20 \mathrm{kpc}$ with a mass similar to the dSphs already discovered, then we would expect to see also a DM annihilation signal from the known dSphs in the Milky Way. Hence, we conclude that a DM origin for these two sources is rejected.

We then applied our model and dSph constraints to the six Fermi-LAT extended unassociated sources recently found in Fermi-LAT Collaboration \& Biteau (2018). We concluded that these too are unlikely to have a DM origin. We could only explain them as coming from DM annihilation if they owe to a DM subhalo with a pre-infall halo mass $M_{200}=10^{9} \mathrm{M}_{\odot}$ and a distance less than $d=3 \mathrm{kpc}$. We are unable to find any such subhalo in the Via Lactea II cosmological simulation. When accounting for the unmodelled effects of tidal stripping and shocking by the Milky Way stellar disc, such subhalos become even rarer. We conclude, therefore, that none of the unassociated, extended, Fermi-LAT sources found to date is likely to have a DM annihilation origin.

Our work represents the first attempt to search for stellar counterparts to Fermi-LAT extended sources that could owe to DM annihilation. The XD method was able to find known star clusters in the fields studied, demonstrating its efficacy. But we were unable to find evidence for any new dSphs in these fields. In future work, we will perform a similar search for stellar counterparts along the line of sight to all Fermi-LAT unassociated sources, including point-like objects.

\section{ACKNOWLEDGEMENT}

IC and DK acknowledge the support of the UK's Science and Technology Facilities Council (STFC Grant ST/K000977/1 and ST/N000811/1). IC is also grateful the STFC Doctoral Training Partnerships Grant (ST/N504488/1). IC thanks the LSSTC Data Science Fellowship Program, where their time as a Fellow has benefited this work. The work of SA was supported in part by JSPS KAKENHI Grants (17H04836, $18 \mathrm{H} 04340$, and $18 \mathrm{H} 04578$ ). JIR would like to acknowledge support from SNF grant PP00P2_128540/1, STFC consolidated grant ST/M000990/1 and the MERAC foundation. CM acknowledges support from grant DGAPA/UNAM IG100115. This work has made use of data from the European Space Agency (ESA) mission Gaia (https://www. cosmos.esa.int/gaia), processed by the Gaia Data Processing and Analysis Consortium (DPAC, https://www. cosmos.esa.int/web/gaia/dpac/consortium). Funding for the DPAC has been provided by national institutions, in particular, the institutions participating in the Gaia Multilateral Agreement. This research has made use of the VizieR catalogue access tool, CDS, Strasbourg, France. The original description of the VizieR service was published in A\&AS $143,23$.

\section{REFERENCES}

Aaronson M., 1983, ApJ, 266, L11

Acero F., et al., 2015, ApJS, 218, 23

Acharya B. S., et al., 2013, Astroparticle Physics, 43, 3

Albert A., et al., 2017, ApJ, 834, 110

Anderson L., Hogg D. W., Leistedt B., Price-Whelan A. M., Bovy J., 2017, preprint, (arXiv:1706.05055)

Antoja T., et al., 2015, MNRAS, 453, 541

Atwood W. B., et al., 2009, ApJ, 697, 1071

Baltz E. A., et al., 2008, J. Cosmology Astropart. Phys., 7, 013 
Bartels R., Ando S., 2015, Phys. Rev. D, 92, 123508

Battaglia G., Helmi A., Breddels M., 2013, New Astron. Rev., 57, 52

Baur J., Palanque-Delabrouille N., Yèche C., Magneville C., Viel M., 2016, J. Cosmology Astropart. Phys., 8, 012

Bechtol K., et al., 2015, ApJ, 807, 50

Belokurov V., et al., 2007, ApJ, 654, 897

Belokurov V., Irwin M. J., Koposov S. E., Evans N. W., GonzalezSolares E., Metcalfe N., Shanks T., 2014, MNRAS, 441, 2124

Bertone G., Hooper D., Silk J., 2005, Phys. Rep., 405, 279

Bertoni B., Hooper D., Linden T., 2015, J. Cosmology Astropart. Phys., 12, 035

Bertoni B., Hooper D., Linden T., 2016, J. Cosmology Astropart. Phys., 5, 049

Bonnivard V., et al., 2015, MNRAS, 453, 849

Bovy J., Hogg D. W., Roweis S. T., 2009, ApJ, 700, 1794

Bovy J., Hogg D. W., Roweis S. T., 2011, Annals of Applied Statistics, 5

Bovy J., et al., 2012, ApJ, 749, 41

Calore F., De Romeri V., Di Mauro M., Donato F., Marinacci F., 2017, Phys. Rev. D, 96, 063009

Calore F., Serpico P. D., Zaldivar B., 2018, preprint, (arXiv: 1803.05508)

Charbonnier A., et al., 2011, MNRAS, 418, 1526

Clowe D., Bradač M., Gonzalez A. H., Markevitch M., Randall S. W., Jones C., Zaritsky D., 2006, ApJ, 648, L109

D’Onghia E., Springel V., Hernquist L., Keres D., 2010, ApJ, 709, 1138

Dias W. S., Alessi B. S., Moitinho A., Lépine J. R. D., 2002, A\&A, 389,871

Diemand J., Kuhlen M., Madau P., 2007, ApJ, 667, 859

Dodelson S., 2011, International Journal of Modern Physics D, 20,2749

Drlica-Wagner A., et al., 2015, ApJ, 813, 109

Evans N. W., Ferrer F., Sarkar S., 2004, Phys. Rev. D, 69, 123501

Fermi-LAT Collaboration Biteau J., 2018, preprint, (arXiv: 1804.08035)

Gaia Collaboration et al., 2016, A\&A, 595, A1

Gaia Collaboration Brown A. G. A., Vallenari A., Prusti T., de Bruijne J. H. J., Babusiaux C., Bailer-Jones C. A. L., 2018, preprint, (arXiv: 1804.09365)

Garrison-Kimmel S., et al., 2017, MNRAS, 471, 1709

Gatto A., Fraternali F., Read J. I., Marinacci F., Lux H., Walch S., 2013, MNRAS, 433, 2749

Geringer-Sameth A., Walker M. G., Koushiappas S. M., Koposov S. E., Belokurov V., Torrealba G., Evans N. W., 2015, Physical Review Letters, 115, 081101

Harvey D., Massey R., Kitching T., Taylor A., Tittley E., 2015, Science, 347, 1462

Hiroshima N., Ando S., Ishiyama T., 2018, preprint, (arXiv: 1803.07691)

Hogg D. W., Blanton M. R., Roweis S. T., Johnston K. V., 2005, ApJ, 629, 268

Holoien T. W.-S., Marshall P. J., Wechsler R. H., 2017, AJ, 153, 249

Hunt J. A. S., Kawata D., Grand R. J. J., Minchev I., Pasetto S., Cropper M., 2015, MNRAS, 450, 2132

Jungman G., Kamionkowski M., Griest K., 1996, Phys. Rep., 267, 195

Kim D., Jerjen H., 2015, ApJ, 808, L39

Kim D., Jerjen H., Mackey D., Da Costa G. S., Milone A. P., 2015, ApJ, 804, L44

Klop N., Zandanel F., Hayashi K., Ando S., 2017, Phys. Rev. D, 95, 123012

Koposov S. E., Belokurov V., Torrealba G., Evans N. W., 2015, ApJ, 805, 130

Laevens B. P. M., et al., 2015a, ApJ, 802, L18

Laevens B. P. M., et al., 2015b, ApJ, 813, 44
Lake G., 1990, Nature, 346, 39

Lindegren L., et al., 2018, preprint, (arXiv:1804.09366)

Luque E., et al., 2016, MNRAS, 458, 603

Luri X., et al., 2018, preprint, (arXiv:1804.09376)

Martin N. F., et al., 2016, MNRAS, 458, L59

Mateo M. L., 1998, ARA\&A, 36, 435

McConnachie A. W., 2012, AJ, 144, 4

Moliné Ã., SÃąnchez-Conde M. A., Palomares-Ruiz S., Prada F., 2017, Mon. Not. Roy. Astron. Soc., 466, 4974

Navarro J. F., Frenk C. S., White S. D. M., 1997, ApJ, 490, 493

Oh S.-H., de Blok W. J. G., Brinks E., Walter F., Kennicutt Jr. R. C., 2011, AJ, 141, 193

Peñarrubia J., Benson A. J., Walker M. G., Gilmore G., McConnachie A. W., Mayer L., 2010, MNRAS, 406, 1290

Planck Collaboration et al., 2016, A\&A, 594, A13

Pryor C., Kormendy J., 1990, AJ, 100, 127

Read J. I., Wilkinson M. I., Evans N. W., Gilmore G., Kleyna J. T., 2006, MNRAS, 367, 387

Read J. I., Iorio G., Agertz O., Fraternali F., 2017, MNRAS, 467, 2019

Rubin V. C., Ford Jr. W. K., Thonnard N., 1980, ApJ, 238, 471

Sawala T., Pihajoki P., Johansson P. H., Frenk C. S., Navarro J. F., Oman K. A., White S. D. M., 2017, MNRAS, 467, 4383

Sharma S., Bland-Hawthorn J., Johnston K. V., Binney J., 2011, ApJ, 730, 3

Silk J., Bloemen H., 1987, ApJ, 313, L47

Skordis C., Mota D. F., Ferreira P. G., Bœhm C., 2006, Physical Review Letters, 96, 011301

Springel V., Frenk C. S., White S. D. M., 2006, Nature, 440, 1137

Steigman G., Dasgupta B., Beacom J. F., 2012, Phys. Rev. D, 86, 023506

Taylor M. B., 2005, in Shopbell P., Britton M., Ebert R., eds, Astronomical Society of the Pacific Conference Series Vol. 347, Astronomical Data Analysis Software and Systems XIV. p. 29

Ural U., Wilkinson M. I., Read J. I., Walker M. G., 2015, Nature Communications, 6, 7599

Winter M., Zaharijas G., Bechtol K., Vandenbroucke J., 2016, Astrophys. J., 832, L6

Xia Z.-Q., et al., 2017, Phys. Rev. D, 95, 102001

This paper has been typeset from a $\mathrm{TE}_{\mathrm{E}} \mathrm{X} / \mathrm{LAT} \mathrm{T}$ file prepared by the author. 